\title{
Sorovares de Leptospira spp. predominantes em exames sorológicos de caninos e humanos no município de Uberlândia, Estado de Minas Gerais
}

\author{
Predominant Leptospira spp. serovars in serological diagnosis of canines and humans in the \\ City of Uberlândia, State of Minas Gerais, Brazil
}

\author{
Jacqueline Ribeiro de Castro ${ }^{1}$, Sandra Renata Sampaio Salaberry ${ }^{2}$, Mariana Assunção de Souza ${ }^{2}$ \\ e Anna Monteiro Correia Lima-Ribeiro ${ }^{3}$
}

\begin{abstract}
RESUMO
Introdução: O objetivo desta pesquisa foi verificar a ocorrência dos principais sorovares de Leptospira spp. em cães domésticos e humanos, notificados no ano de 2008, bem como os principais fatores de riscos em uma abordagem geográfica relacionados à doença no município de Uberlândia, Estado de Minas Gerais, Brasil. Métodos: Foram examinadas 268 amostras de soro sanguíneo de cães de diferentes bairros pertencentes aos distritos sanitários norte, sul, leste, oeste e central deste município, colhidas durante a campanha de vacinação antirrábica animal, em agosto de 2008. Foi realizada uma abordagem geográfica do município e avaliada a localização de áreas periféricas, aterro sanitário, coleta de lixo, notificação de roedores, casos de leptospirose humana e áreas de alagamento decorrente de enchentes, durante o ano de 2008. A leptospirose foi diagnosticada pela técnica de soroaglutinação microscópica (SAM), padrão-ouro para diagnóstico da leptospirose animal e humana. Resultados: Os cães reagiram principalmente aos sorovares Autumnalis (34,2\%) e Tarassovi (23,7\%), sendo este, também detectado em humanos em 2008 A ocorrência destes sorovares pode estar relacionada com uma fonte de infecção comum as duas espécies, ou a hipótese de que o cão possa ser a fonte de infecção para o ser humano. $\mathrm{O}$ distrito sanitário leste apresentou um maior número de cães reagentes. Conclusões: A leptospirose ocorreu nos cães e humanos no município de Uberlândia no ano de 2008. Esta doença muitas vezes negligenciada deve ser prevenida por representar risco à saúde pública e se parecer com outras doenças também endêmicas como a dengue.
\end{abstract}

Palavras-chaves: Zoonose. Leptospirose. Soroprevalência.

\begin{abstract}
Introduction: This study aimed to verify the occurrence of the principal Leptospira spp. serovars in domestic dogs and humans, notified in 2008, and the main risk factors in a geographic approach to the disease in the City of Uberlândia, State of Minas Gerais, Brazil. Methods: Canine blood serum samples $(n=268)$ from different districts, belonging to the Northern, Southern, Eastern, Western and Central Sanitary Districts of Uberlandia, were collected during an animal vaccination campaign against rabies, in August 2008. A geographic approach to the city was conducted, including evaluation of locations in peripheral areas, waste landfills, garbage collection, rodent notification, human leptospirosis cases and overflow flood areas, during 2008. Leptospirosis was diagnosed using the microscopic agglutination test (MAT), the gold standard for anima and human leptospirosis diagnosis. Results: Dogs mainly reacted to Autumnalis (34.2\%) and Tarassovi (23.7\%) serovars, while in humans, predominance of Tarassovi serovars occurred in the cases registered. The occurrence of these serovars could be related to an infection source common to both species, or dogs could be the infection source for humans. The Eastern Sanitary District showed a greater number of reactive dogs. Conclusions: Leptospirosis occurred in dogs and humans of the City of Uberlandia in 2008. This often neglected disease must be prevented because represents a public health risk and resembles other endemic illness like dengue.
\end{abstract}

Keywords: Zoonosis. Leptospirosis. Seroprevalence.

1. Programa de Pós-Graduação, Faculdade de Medicina Veterinária, Universidade Federal de Uberlândia, Uberlândia, MG. 2. Faculdade de Medicina Veterinária, Universidade Federal de Uberlândia, Uberlândia, MG. 3. Disciplina de Doenças Bacterianas dos Animais Domésticos, Faculdade de Medicina Veterinária, Universidade Federal de Uberlândia, Uberlândia, MG.

Endereço para correspondência: Ms. Jacqueline Ribeiro de Castro. Lab. de Doenças Infectocontagiosas/ FAMEV/UFU. Av. Ceará s/no, Bloco 2D, Sala 33, Campus Umuarama, 38400-902 Uberlândia, MG.

Tel: 5534 3218-2231; Fax: 5534 3218-2612

e-mail: jack_ufu@yahoo.com.br

Recebido para publicação em 30/08/2010

Aceito em 16/11/2010

\section{INTRODUÇÃO}

A leptospirose é uma doença infectocontagiosa de distribuição mundial, caracterizada por ser uma zoonose que acomete animais domésticos, silvestres e a espécie humana ${ }^{1}$. O rato de esgoto (Rattus novergicus) e os cães são os principais reservatórios da leptospirose no ambiente urbano ${ }^{2}$, portanto, sendo importantes na transmissão da doença para a espécie humana.Esta doençaémais comum emáreas próximas às favelas ${ }^{3}$, pois normalmente nestes locais há falta de saneamento básico levando uma maior exposição das populações humana e canina aos roedores.

Melhorias nas condições de moradia, com instalação de redes de esgoto, drenagem de áreas alagadas e um sistema de coleta de lixo eficiente são importantes medidas a serem adotadas para diminuição da leptospirose na população humana ${ }^{4}$. A vacinação dos animais susceptíveis mostra-se como uma forma bastante eficiente para o controle da doença nos mesmos e, consequentemente, em seres humanos.

A doença tem maior prevalência nas regiões de clima tropical, quente e úmido e em locais de pobres condições sanitárias ${ }^{5}$. No Brasil, a zoonose é endêmica, com a maior parte dos casos ocorrendo nas épocas de maior precipitação pluviométrica. No país, a manutenção do agente no meio é favorecida pela vasta população de roedores e pelo clima tropical úmido.

Os sorovares mais prevalentes em pesquisa que determinou a situação da leptospirose canina no Brasil foram Canicola, Copenhageni, Icterohaemorrhagiae seguido pelo Autumnalis ${ }^{6}$. A média das prevalências encontradas em todo país ${ }^{7}$ foi de $26 \%$.

Os roedores são considerados os principais reservatórios da infecção. Cogita-se que os sorovares presentes nos roedores em um determinado local são semelhantes aos presentes em cães que vivem nesse mesmo ambiente, fato este que foi discutido em pesquisa realizada em Trinidade na Índia ${ }^{8}$ a qual apontou o sorovar Copenhageni predominante em ambas as espécies. 
Importância especial deve ser conferida à espécie Rattus norvegicus, conhecida como ratazana de esgotos, principal espécie transmissora em centros urbanos. Sua proliferação é verificada em grandes cidades, onde as redes pluviais e de esgoto não recebem tratamento adequado e, com frequência, se conectam possibilitando maior contaminação ambiental ${ }^{9,10}$.

O cão tem papel importante na transmissão da doença ao homem, por manter a leptospira por longo período nos rins, podendo eliminá-la na urina sem apresentar sinais clínicos ou após obter melhora clínica. Esse fato se torna mais agravante devido aos hábitos domésticos desses animais e sua estreita relação com os humanos ${ }^{5,11}$

Cães são boas sentinelas para detectar a presença de leptospirose no ambiente ${ }^{12}$ e são fatores chave para o entendimento da epidemiologia da doença, sendo importante o diagnóstico e tratamento dos animais doentes a fim de evitar a infecção humana ${ }^{13}$.

A incidência da leptospirose humana tem crescido em países da América Latina associada aos desastres naturais e ao crescimento desordenado das cidades ${ }^{14}$. No Brasil, em 2008, de acordo com o Ministério da Saúde foram registrados 3.306 casos, sendo 949 na região sudeste e 63 no Estado de Minas Gerais. Segundo a Vigilância Epidemiológica da Prefeitura Municipal de Uberlândia, no ano de 2008, foram registrados no município 11 casos suspeitos e dois casos confirmados de leptospirose humana.

A presente pesquisa objetivou investigar a ocorrência dos principais sorovares de Leptospira spp. em cães domésticos e humanos notificados no ano de 2008, bem como os principais fatores de riscos em uma abordagem geográfica e epidemiológica relacionados à doença no município de Uberlândia, Estado de Minas Gerais.

\section{MÉTODOS}

A pesquisa foi realizada no município de Uberlândia situado no Triângulo Mineiro, Estado de Minas Gerais, Brasil, limitado pelas coordenadas geográficas $18^{\circ} 30^{\prime}$ e 19³0' de latitude sul e 47 50’ e $48^{\circ} 50^{\prime}$ de longitude oeste com uma área de $4115,09 \mathrm{~km}^{2}$. Apresenta clima tropical com pluviosidade anual de $1.500 \mathrm{~mm}$ e temperatura média de $22^{\circ} \mathrm{C}^{15}$.

O experimento foi conduzido durante a 26 Campanha de Vacinação Antirrábica Animal, etapa urbana, em agosto de 2008. O dimensionamento da amostra foi determinado com base na prevalência esperada de $26 \%$, a qual corresponde à prevalência média da leptospirose canina no Brasil ${ }^{7}$, totalizando 268 cães.

A colheita de sangue dos cães foi realizada em 60, dos 300 postos fixos de vacinação distribuídos nos cinco distritos sanitários do município: central, norte, leste, oeste e sul. Após prévia vacinação antirrábica e autorização dos proprietários, foram selecionados aleatoriamente cinco cães por posto da campanha.

Para realização do exame sorológico de leptospirose foram colhidos $5 \mathrm{~mL}$ de sangue por meio da veia cefálica acessória, com seringas de $5 \mathrm{~mL}$ e agulha $25 \mathrm{x} 7$ estéreis, após antissepsia prévia com álcool. As amostras foram acondicionadas em tubos sem anticoagulante para extração do soro e mantidas refrigeradas $\left(2\right.$ a $\left.8^{\circ} \mathrm{C}\right)$. O material foi transportado ao Laboratório de Doenças Infectocontagiosas da Faculdade de Medicina Veterinária da Universidade Federal de Uberlândia (FAMEV-UFU) e centrifugado a $2.500 \mathrm{rpm}$, durante $5 \mathrm{~min}$. Em seguida, as amostras de soro foram acondicionadas em microtubos de polietileno de fundo cônico e congeladas a $-20^{\circ} \mathrm{C}$ até a realização da SAM.

As análises sorológicas foram processadas por meio do teste de soroaglutinação microscópica (SAM $)^{3,16}$, padrão ouro no diagnóstico da leptospirose humana e animal, padronizado pelo Ministério da Saúde, com uma coleção de 12 antígenos vivos que incluíram os sorovares Autumnalis, Australis, Bataviae, Bratislava, Canicola, Grippotyphosa, Hardjo, Hebdomadis, Icterohaemorrhagiae, Pomona, Tarassovi e Wolffi.

Os antígenos foram preparados a partir de matrizes mantidas Laboratório de Doenças Infectocontagiosas da UFU, repicadas semanalmente em meio de cultura EMJH $\left(\right.$ Difco $\left.^{\circledR}\right)$, enriquecido com $10 \%$ de soro de coelho, mantido em estufa a $30^{\circ} \mathrm{C}$ e utilizados próximo ao terceiro dia de incubação, livre de contaminação e de autoaglutinação. Para determinação dos cães sororreagentes, foi utilizada a diluição de 1:100, considerando-se amostras reagentes, as quais apresentaram aglutinação igual ou superior a $50 \%{ }^{16}$.

Casos de leptospirose humana foram determinados a partir de coleta de dados oficiais junto aos registros da Vigilância Epidemiológica da Prefeitura Municipal (Vigep) de Uberlândia (PMU), registros de exames humanos de SAM realizados no Laboratório de Doenças Infectocontagiosas da Faculdade de Medicina Veterinária da Universidade Federal de Uberlândia, Estado de Minas Gerais, e laboratórios da rede particular do município pesquisado. Por se tratar de um estudo em cães assintomáticos, domiciliados e, possivelmente portadores crônicos, foram considerados os casos humanos confirmados no ano anterior (2007) e posterior (2009) a realização desta pesquisa.

De acordo com a Vigep da PMU seguindo as normas do Ministério da Saúde ${ }^{16}$ foram considerados casos suspeitos todos aqueles que tiveram suspeita clínica da doença e amostras de soro enviadas para a realização da SAM. Após a realização do exame sorológico foram classificados como negativo os casos não reagentes ao SAM e positivos todos aqueles se mostraram reagentes na SAM, exame sorológico padrão na confirmação da leptospirose humana.

Foi realizada uma abordagem geográfica do município com coleta de dados dos arquivos da PMU do Banco de dados Integrados de 2008, Vigilância Epidemiológica e Centro de Controle de Zoonoses (CCZ). Avaliou-se por distrito sanitário o saneamento ambiental com relação à localização de áreas periféricas com baixa infraestrutura, aterro sanitário, coleta de lixo, notificação de roedores e áreas de alagamento decorrente de enchentes durante o ano de 2008. Estes dados foram correlacionados com os distritos sanitários que apresentaram cães reagentes contra Leptospira spp.

\section{Considerações éticas}

O experimento foi conduzido de acordo com normas e aprovação (015/08) do Comitê de Ética na Utilização de Animais da Universidade Federal de Uberlândia.

\section{RESULTADOS}

Das 268 amostras de soro sanguíneo analisadas, 76 apresentaram reagentes à SAM, obtendo-se uma ocorrência de 28,4\%. Os sorovares de maior frequência foram o Autumnalis (34,2\%), Tarassovi $(23,7 \%)$ seguido pela sorovariedade Canicola $(17,1 \%)$ e Grippotyphosa (14,5\%), demonstrados na Tabela 1. Não foram detectados cães sororreagentes aos sorovares Hebdomadis, Bataviae e Hardjo. 
TABELA 1 - Frequência de sorovares em 76 amostras de soro de cães reagentes domiciliados em Uberlândia, Estado de Minas Gerais, no ano de 2008.

\begin{tabular}{lcc}
\hline Sorovar & Cães sororreagentes & Frequência relativa \\
\hline Autumnalis & FA & $\%$ \\
\hline Tarassovi & 26 & 34,2 \\
\hline Canicola & 18 & 23,7 \\
\hline Grippotyphosa & 13 & 17,1 \\
\hline Bratislava & 11 & 14,5 \\
\hline Icterohaemorragiae & 3 & 3,9 \\
\hline Australis & 2 & 2,7 \\
\hline Pomona & 1 & 1,3 \\
\hline Wolffi & 1 & 1,3 \\
\hline Total & 1 & 1,3 \\
\hline FA. frequência absoluta & $\mathbf{7 6}$ & $\mathbf{1 0 0 , 0}$
\end{tabular}

FA: frequência absoluta

TABELA 2 - Coaglutinação de diferentes sorovares de Leptospira spp. em cães domiciliados no município de Uberlândia, Estado de Minas Gerais, no ano de 2008.

\begin{tabular}{lcc}
\hline Sorovares & $\begin{array}{c}\text { Frequência } \\
\text { absoluta }\end{array}$ & $\begin{array}{c}\text { Frequência relativa } \\
\%\end{array}$ \\
\hline Autumnalis+Brastislava + Pomona & 2 & 13,3 \\
\hline Autumnalis+Tarassovi & 2 & 13,3 \\
\hline Autumnalis+ Grippotyphosa & 2 & 13,3 \\
\hline Autumnalis+ Icterohaemorrhagiae & 2 & 13,3 \\
\hline Autumnalis+ Pomona & 1 & 6,7 \\
\hline Autumnalis+Brastilava & 1 & 6,7 \\
\hline Autumnalis+Canicola & 1 & 6,7 \\
\hline Brastilava+Canicola & 1 & 6,7 \\
\hline Brastilava+Wolffi & 1 & 6,7 \\
\hline Canicola+Grippotyphosa+Tarassovi & 1 & 6,7 \\
\hline Canicola+Tarassovi & 1 & 6,7 \\
\hline Total & $\mathbf{1 5}$ & $\mathbf{1 0 0 , 0}$ \\
\hline
\end{tabular}

Dos cães sororreagentes, 15 (19,7\%) apresentaram anticorpos para mais de um sorovar de Leptospira spp. (Tabela 2). Nesses casos de coaglutinação, considerou-se reagente a sorovariedade de maior titulação.

O distrito sanitário leste (35\%) apresentou um maior percentual de cães reagentes seguido pelo sul (26\%), oeste (22\%), norte (16\%) e central com apenas $1 \%$.

De acordo com os dados registrados pelo CCZ da PMU, no ano de 2008, o maior número de notificações de ocorrência de roedores e solicitação de serviço de controle foi identificado no distrito sanitário Norte, seguido pelo leste (Tabela 3), no qual se verificou um maior número de cães que apresentaram anticorpos anti-Leptospira spp.

Os resultados e registros de notificação de casos humanos e sorovares ocorridos de 2007 a 2009 encontram-se descritos nas Tabelas 4 e 5.

TABELA 3 - Notificações de roedores de acordo com os distritos sanitários de Uberlândia, Estado de Minas Gerais, no ano de 2008.

\begin{tabular}{lc}
\hline Distritos sanitários & Notificações \\
\hline Norte & 353 \\
\hline Leste & 342 \\
\hline Oeste & 319 \\
\hline Sul & 270 \\
\hline Central & 269
\end{tabular}

Fonte: Centro de Controle de Zoonoses (CCZ) da Prefeitura Municipal de Uberlândia.

TABELA 4 - Casos de leptospirose humana em Uberlândia, Estado de Minas Gerais, classificados conforme ano de notificação.

\begin{tabular}{lccc}
\hline & \multicolumn{3}{c}{ Ano } \\
\cline { 2 - 4 } Classificação final & 2007 & $2008^{*}$ & 2009 \\
\hline Aguarda resultado & 0 & 0 & 2 \\
\hline Confirmado & 0 & $\mathbf{2}$ & 0 \\
\hline Descartado & 8 & 12 & 12 \\
\hline Total & $\mathbf{8}$ & $\mathbf{1 4}$ & $\mathbf{1 4}$
\end{tabular}

Fonte: Vigilância Epidemiológica do Sistema de Informação de Agravos de Notificação (SINAN) dos anos 2007, 2008 e 2009, Uberlândia, MG. ${ }^{*}$ Ano de estudo da pesquisa.

TABELA 5 - Resultados da SAM* de leptospirose humana realizados no Laboratório de Doenças Infectocontagiosas da FAMEV, UFU e Laboratórios particulares do município de Uberlândia, Estado de Minas Gerais, no período de 2007 a 2009.

\begin{tabular}{|c|c|c|c|c|c|c|}
\hline \multirow{2}{*}{$\begin{array}{l}\text { Exames } \\
\text { registrados }\end{array}$} & \multicolumn{3}{|c|}{ Laboratório UFU ${ }^{\mathrm{a}}$} & \multicolumn{3}{|c|}{ Laboratório particular ${ }^{b}$} \\
\hline & 2007 & $2008^{* *}$ & 2009 & 2007 & $2008^{* *}$ & 2009 \\
\hline Suspeitos & 5 & 11 & 7 & 10 & 8 & 9 \\
\hline Negativos & 3 & 9 & 6 & 10 & 6 & 9 \\
\hline Positivos & 2 & 2 & 1 & 0 & 2 & 0 \\
\hline Sorovares & $\begin{array}{c}\text { Canicola, } \\
\text { Hardjo }\end{array}$ & Tarassovi & $\begin{array}{c}\text { Wolffi, } \\
\text { Grippotyphosa }\end{array}$ & - & $\begin{array}{c}\text { Bataviae, Canicola, } \\
\text { Copenhageni, Djasiman, } \\
\text { Icterohaemorrhagiae, } \\
\text { Panama, Patoc, } \\
\text { Tarassovi, Wolff }\end{array}$ & - \\
\hline
\end{tabular}

Fonte: aArquivos do Laboratório de Doenças Infectocontagiosas da Faculdade de Medicina Veterinária (FAMEV) da Universidade Federal de Minas Gerais (UFU) nos anos de 2007, 2008 e 2009, Uberlândia, MG. bArquivos de Laboratórios Particulares - Divisão Humana, Uberlândia, MG, no período de 2007, 2008 e 2009.

*SAM - Técnica de soroaglutinação microscópica em campo escuro. ${ }^{* *}$ Ano de estudo da pesquisa. 


\section{DIscussão}

A ocorrência da doença neste percentual elevado em cães demonstra que a leptospirose é uma doença comum e provavelmente subestimada devido à grande quantidade de animais assintomáticos, embora cronicamente afetados.

Os casos de coaglutinação podem ser explicados devido exposição a mais de um sorovar, à diversidade de reservatórios e ambientes aos quais os animais se expõem ${ }^{17}$.

Em estudo realizado em Belo Horizonte, $\mathrm{MG}^{3}$, a prevalência encontrada foi de $13,1 \%$ em cães recolhidos pelo Centro de Controle de Zoonoses oriundos de domicílios e cães errantes. Já em Patos, $\mathrm{PB}^{1}$, encontraram uma soroprevalência de $20 \%$, no entanto, avaliaram apenas cães errantes.

Comparando os resultados da presente investigação com outras conduzidas em diferentes cidades do país, verifica-se que o percentual de cães reagentes encontrados $(28,4 \%)$ foi superior ao encontrado no município de Campina Grande ${ }^{11}$, PB $(21,4 \%)$ e em Botucatu ${ }^{18}$, SP $(17,9 \%)$. Estas pesquisas também foram realizadas durante a campanha de vacinação antirrábica com seleção aleatória dos animais pertencentes a diferentes localidades.

Em Pelotas, $\mathrm{RS}^{19}$ e Londrina, $\mathrm{PR}^{20}$, encontraram prevalências superiores, $34,8 \%$ e $30,5 \%$, respectivamente. Isto, possivelmente, se deve ao fato dos autores terem utilizados em suas pesquisas amostras de cães com suspeita clínica de leptospirose.

A maior incidência do sorovar Autumnalis (34,2\%) também foi identificada em outros estudos ${ }^{1,21}$ os quais apresentaram $20 \%$ e $22,2 \%$, respectivamente, de cães sororreagentes frente a esta sorovariedade. $\mathrm{O}$ aparecimento de sorovares incomuns pode ser explicado pelo fato do compartilhamento do ambiente por diferentes espécies animais, o que pode promover a associação e adaptação de agentes a novos hospedeiros ${ }^{21}$.

Observou-se baixa ocorrência da doença na zona central do município, o que pode estar relacionada com maior efetividade no saneamento básico, com coleta de lixo diária e tratamento de água e esgoto em sua total extensão por ser considerada parte nobre do município. Os cães do distrito sanitário leste apresentaram um maior número de cães reagentes na SAM. Neste distrito, concentram-se alguns bairros com déficit estrutural e baixo índice de urbanização, tendo um elevado número de terrenos baldios e setor de chácaras.

A leptospirose detectada em humanos de Belo Horizonte, MG, ocorreu principalmente nas áreas de bolsões de pobreza, com grande densidade populacional e carência de infra-estrutura ${ }^{22}$. Na distribuição espacial, a doença esteve presente em maior percentual em áreas com maior concentração de redes fluviais. Dentre os indivíduos avaliados, $12 \%$ tiveram contato com a água ou animais contaminados, demonstrando assim, a importância do cão na cadeia epidemiológica da doença na zona urbana.

De acordo com a Defesa Civil da PMU, MG, as áreas de emergência pluviométricas estão distribuídas por todas as bacias de córregos e rio que passam pelo município. No ano de 2008, houve registros de emergências pluviométricas com precipitações elevadas em locais como Av. Governador Rondon Pacheco, Av. Minervina Cândida e Av. Getúlio Vargas. No entanto, estas são vias urbanas que ligam os principais pontos de Uberlândia, atravessam o município e geograficamente pertencem a vários bairros e a mais de um distrito sanitário, não sendo possível correlacionar com os casos de leptospirose canina.
Alguns locais se destacaram nesta avaliação, como o bairro Morumbi localizado no distrito sanitário leste de maior ocorrência de cães reagentes. Este bairro foi alagado em 2008 diversas vezes no período chuvoso em situação crítica havendo a necessidade de implementar rotas alternativas para acesso ao bairro até regularização do nível de água.

A ocorrência de enchentes é considerada um fator de risco para a leptospirose canina e humana, pois durante as épocas chuvosas ocorre maior facilidade de disseminação do agente, eliminado pela urina de roedores e outros reservatórios ${ }^{11,23}$.

A presença de áreas alagadiças próximas às residências é considerada um fator de risco para a leptospirose canina ${ }^{20}$. É importante limitar o acesso dos cães a áreas pantanosas, lamacentas, lagos e áreas alagadiças como medida de prevenção da doença ${ }^{6}$.

Foi observado também que a região central teve um menor número de registros de notificações de presença de roedores e foi o distrito com um menor percentual de cães reagentes contra Leptospira spp. enquanto, os distritos sanitários norte e leste (Tabela 3) apresentaram um maior número de notificações de roedores, sendo que este se verificou um maior número de cães que apresentaram anticorpos anti-Leptospira spp.

Os roedores são considerados hospedeiros de manutenção de diversos sorovares e contribuem para a contaminação ambiental permitindo assim a possível infecção do cão e do ser humano. O contato dos cães com roedores constitui um fator de risco, uma vez que, a presença de ratos está intimamente ligada ao aparecimento da doenç $\mathrm{a}^{24}$.

A coleta de lixo no município de Uberlândia tem sido realizada diariamente no distrito sanitário central e três vezes por semana nos demais distritos. $\mathrm{O}$ aterro sanitário localiza-se no setor norte e neste verificou-se um maior número de notificações de roedores, enquanto, no central observou-se um menor registro de roedores e de cães reagentes contra Leptospira spp.

A incidência da leptospirose humana tem elevado na América Latina devido ao crescimento desordenado das cidades e aos desastres naturais ${ }^{14}$.

De acordo com dados da Vigilância Epidemiológica da Prefeitura Municipal de Uberlândia, no ano de 2008, foram notificados apenas dois casos confirmados de leptospirose humana, sendo um proveniente da área rural e outro provindo do distrito sanitário oeste da zona urbana do município. Estes dados podem estar aquém da realidade devido à falta de confirmação, e notificação dos casos humanos. Acredita-se ainda, que a semelhança entre a sintomatologia da leptospirose humana e outras doenças tropicais como a dengue, possa explicar a discrepância entre os dados registrados em cães e seres humanos nessa pesquisa. Em algumas épocas do ano de 2008 a dengue se apresentou de forma epidêmica neste município de acordo com dados da Vigep.

A rapidez que a clínica exige do profissional de posto de saúde pode levar ao negligenciamento no correto diagnóstico de leptospirose.

Tal fato pode ser sustentado pela avaliação feita nos registros do Laboratório de Doenças Infectocontagiosas da UFU e de laboratórios particulares. Em um levantamento realizado de exames sorológicos de SAM de leptospirose humana, realizados nos últimos três anos (2007 a 2009), ao serem confrontados com os dados oficiais de notificação (Vigep), verificou-se um número maior de casos registrados confirmados de pacientes provindos do município de Uberlândia, MG, neste mesmo período, conforme Tabelas 4 e 5. 
No ano de 2008, foram identificados nos exames sorológicos humanos, pacientes positivos para leptospirose, reagentes a vários sorovares, incluindo o sorovar Tarassovi (Tabelas 4 e 5), o qual foi o segundo sorovar mais prevalente na população canina avaliada. A ocorrência deste sorovar pode estar relacionada com uma fonte de infecção comum as duas espécies. Discute-se a hipótese de que o cão possa ser a fonte de infecção para o ser humano. Entre os animais domésticos, em ambiente urbano, a principal fonte de infecção da leptospirose humana são os cães. Esses animais vivem em contato direto com os seres humanos e podem eliminar leptospiras vivas pela urina durante meses, mesmo sem apresentar nenhum sinal clínico ${ }^{25}$.

Há indícios que o cão tem potencial de ser hospedeiro de manutenção para a espécie humana, mediante a realização do primeiro estudo ${ }^{26}$ que explorou a detecção molecular do sorovar Wolffii em ambos hospedeiros humanos e animais (cães e ovinos) e determinaram que o fragmento amplificado foi $100 \%$ semelhante nas espécies estudadas evidenciando que estes animais hospedeiros inclusive a espécie canina podem ter o sorovar Wolffii circulando e sendo transmitido ao ser humano. Porém, diferentemente da pesquisa realizada na Argentina ${ }^{27}$ que determinou as características clínicas e os fatores de risco para a leptospirose humana e não encontrou uma correlação significativa da ocorrência da doença em humanos com a presença de cães. No entanto, apontou como variáveis significantes, idade maior que 30 anos, ocupação no setor rural, contato com água de superfície contaminada e contato com inundações.

As áreas de maior risco de leptospirose em cães na Cidade de Belo Horizonte, MG, coincidiram com as regiões de vila, favelas e bairros da periferia, os quais possuíam deficiência de saneamento ambiental ${ }^{3}$.

As áreas periféricas de Uberlândia localizam-se na região leste do município, consideradas áreas de invasão e que, atualmente, se emanciparam como bairros, como Dom Almir, Prosperidade, Joana Darc e São Francisco, no entorno do bairro Morumbi, e apresentam características de assentamentos urbanos com loteamentos irregulares, condições insalubres com infraestrutura precária, falta de saneamento e urbanização inadequada, o que ocasiona grandes impactos para a saúde humana ${ }^{28}$.

No presente estudo, o distrito sanitário leste também foi o que apresentou maior percentual de cães reagentes contra Leptospira spp. Neste local, observaram-se, em maiores proporções, conglomerados de áreas periféricas e bairros com déficits infraestruturais, com deficiência no saneamento básico de água e esgoto. O bairro Tibery localizado neste distrito apresentou seis cães reagentes, sendo o bairro com maior ocorrência deste estudo.

Vale salientar que os cães deste estudo eram domiciliados e não apresentavam sintomatologia clínica da doença e que provavelmente albergavam a bactéria de forma assintomática, servindo como reservatórios da doença, podendo infectar o ser humano e manter a cadeia epidemiológica do agente. Fatos importantes sob o ponto de vista epidemiológico, pois atualmente o cão de estimação já representa um membro de grande número de famílias do Brasil e do mundo.

Uma ocorrência de $28,4 \%$ de cães reagentes foi detectada em cães domiciliados do município de Uberlândia, MG. Os sorovares mais frequentes nos cães foram o Autumnalis (34,2\%) seguido por Tarassovi (23,7\%), sendo este, também detectado em humanos em 2008. O distrito sanitário leste apresentou em maior número de cães regentes contra Leptospira spp. Novos estudos com maior casuística de casos humanos devem ser realizados para determinar a correlação existente entre a leptospirose canina e humana.

\section{AGRADECIMENTOS}

A todos da Prefeitura Municipal e ao Centro de Controle de Zoonoses de Uberlândia, MG que gentilmente nos receberam e forneceram dados essenciais e aos técnicos do Laboratório de Doenças Infecto-contagiosas da Faculdade de Medicina Veterinária da Universidade Federal de Uberlândia.

\section{CONFLITO DE INTERESSE}

Os autores declaram não haver nenhum tipo de conflito de interesse no desenvolvimento do estudo.

\section{SUPORTE FINANCEIRO}

Coordenação de Aperfeiçoamento de Pessoal de Nível Superior (CAPES).

\section{REFERÊNCIAS}

1. Batista CSA, Azevedo SS, Alves CJ, Vasconcellos AS, Morais M, Clementino IJ, et al. Soroprevalência de leptospirose em cães errantes da cidade de Patos, Estado da Paraíba, Brasil. Braz J Vet Res Anim Sci 2004; 41:131-136.

2. Ribeiro MG, Beloni SN, Langoni H, Silva AV. Leptospirose canina. Boletim Técnico. Campinas: Departamento Técnico Fort Dodge Saúde Animal; 2003.

3. Magalhães DF, Silva JÁ, Moreira EC, Wilke VML, Haddad JPA, Meneses JNC Prevalência de aglutininas anti-Leptospira interrogans em cães de Belo Horizonte, Minas Gerais, 2001 a 2002. Arq Bras Med Vet Zootec 2006; 58:167-174.

4. Jaszczerski DCFC. Cinética da resposta imune humoral em cães imunizados com Leptospira interrogans sorovares icterohaemorragiae, canicola, pomona e grippotyphosa. [Dissertação Mestrado]. [Curitiba (PR)]: Universidade Federal do Paraná; 2005. 82p.

5. Brown K, Prescott J. Leptospirosis in the family dogs: a public health perspective. Can Med Assoc J 2008; 178:399-401.

6. Mello LPP, Manhoso FFR. Aspectos epidemiológicos da leptospirose canina no Brasil. Unimar Cienc 2007; 16:27-32.

7. Boechat JUD, Machado JP. Prevalência da leptospirose canina no Brasil. Vet Ser 2004; $1: 40-47$

8. Suepaul SM, Carrington CVF, Campbell M, Borde G, Adesiyun AA. Serovars of Leptospira isolated from dogs and rodents. Epidemiol Infect 2010; 138:1059-1070.

9. Possas CA. Urbanização, ecologia e emergência de formas graves da leptospirose: análise comparativa de dados secundários nacionais. Rio de Janeiro: Anais do evento comemorativo do centenário do Instituto Oswaldo Cruz e da Fundação Oswaldo Cruz; 2000.

10. Sarkar V, Nascimento SF, Barbosa R, Martins R, Nuevo H, Kalafanos I, et al Population-Based Case-control investigation of risk factors for leptospirosis during in Urban Epidemic. Am J Trop Med Hyg 2002; 66:605-610.

11. Batista CSA, Alves CJ, Azevedo SS, Vasconcellos AS, Morais ZM, Clememtino IJ, et al. Soroprevalência e fatores de risco para a leptospirose em cães de Campina Grande, Paraíba. Arq Bras Med Vet Zootec 2005; 57:179-185.

12. Ghneim GS, Viers JH, Chomel BB, Kass PH, Descollonges DA, Johnson ML Use of a case-control study and geographic information systems to determine environmental and demographic risk factors for canine leptospirosis. Vet Res 2007; 38:37-50.

13. Oliveira ST. Leptospirose canina: dados clínicos, laboratoriais e terapêuticos em cães naturalmente infectados. [Tese Doutorado]. [Porto Alegre (RS)] Universidade Federal do Rio Grande do Sul; 2010. 89p.

14. Oliveira DSC, Guimarães MJB, Medeiros Z. Modelo produtivo para a leptospirose. Rev Patol Trop 2009; 38:17-26.

15. Brito J, Prudente TD. Mapeamento do uso da terra e cobertura vegetal do município de Uberlândia-MG, utilizando imagens ccd/cbers2. Cam Geogr 2005 ; $13: 144-153$. 
16. Ministério da Saúde. Manual de Leptospirose. 2a ed. rev. Brasília: Fundação Nacional de Saúde. Centro Nacional de Epidemiologia. Coordenação de Controle de Zoonoses e Animais Peçonhentos. Programa Nacional de Leptospirose; 1995.

17. Barwick RS, Mohammed HO, Mcdonough PL, White ME. Epidemiologic features of equine Leptospira interrogans of human significance. Prev Vet Med 1998; 36:153-165.

18. Silva WB, Simões LB, Lopes ALS, Padovani CR, Langoni H, ModoloJR. Avaliação de fatores de risco de cães sororeagentes à Leptospira spp e sua distribuição espacial, em área territorial urbana. Braz J Vet Res Anim Sci 2006; 43:783-792.

19. Ávila MO, Furtado LRI, Teixeira MM, Rosado RLI, Martins LFS, Brod CS. Aglutininas anti-leptospíricas em cães na área de influencia do Centro de Controle de Zoonoses, Pelotas, RS, Brasil, no ano de 1995. Cienc Rural 1998; 28: 107-110.

20. Querino AMV, Delbem ACB, Oliveira RC, Silva FG, Muller EE, Freire RL, et al. Fatores de risco associados à leptospirose em cães do município de Londrina-PR. Semin Cienc Agrar 2003; 24:27-34.

21. Aguiar DM, Cavalcante GT, Marvulo MFV, Silva JCR, Pinter A, Vanconcellos SA, et al. Fatores de risco associados à ocorrência de anticorpos anti-Leptospira spp. Em cães do município de Monte Negro, Rondônia, Amazônia Ocidental Brasileira. Arq Bras Med Vet Zootec 2007; 59:70-76.

22. Figueiredo CM, Mourão AC, Oliveira MAA, Alves WR, Oteman MC, Chamone $\mathrm{CB}$, et al. Leptospirose humana no município de Belo Horizonte, Minas Gerais, Brasil: uma abordagem geográfica. Rev Soc Bras Med Trop 2001; 34:331-338.

23. Mascolli R, Pinheiro SR, Vasconcellos AS, Ferreira F, Morais ZM, Pinto CO, et al. Inquérito sorológico para leptospirose em cães do município de Santana de Paraíba, São Paulo, utilizando a campanha de vacinação anti-rábica do ano de 1999. Arq Inst Biol 2002; 69:25-32.

24. Lopes ALS, Silva WB, Padovani CR, Langoni H, Modolo JR. Frequência sorológica antileptospírica em cães: sua correlação com roedores e fatores ambientais, em área territorial urbana. Arq Inst Biol 2005; 72:289-296.

25. Faine S, Adler B, Bolin C, Perolat P. Leptospira and leptospirosis. $2^{\mathrm{a}}$ ed. Melbourne: MedSci; 1999.

26. Zakeri S, Khorami N, Ganji ZF, Sepahian N, Malmasi AA, Gouya MM, et al. Leptospira wolffi, a potential new pathogenic Leptospira species detected in human, sheep and dog. Infect Genet Evol 2010; 10:273-277.

27. Vanasco NB, Schmeling MF, Lottersberger J, Costa F, Ko AI, Tarabla HD. Clinical characteristics and risk factors of human leptospirosis in Argentina (1999-2005). Acta Tropica 2008; 107:255-258.

28. Ramires JCL, Santos MAF. Exclusão social em Uberlândia: algumas reflexões a partir do bairro Dom Almir e seu entorno. Cam Geogr 2001; 2:73-87. 